\title{
La culture de la réalité virtuelle
}

\author{
Jacques Coulardeau
}

\section{OpenEdition}

Journals

Édition électronique

URL : http://journals.openedition.org/asp/4045

DOI : 10.4000/asp.4045

ISSN : 2108-6354

\section{Éditeur}

Groupe d'étude et de recherche en anglais de spécialité

\section{Édition imprimée}

Date de publication : 1 décembre 1994

Pagination : 111-120

ISSN : 1246-8185

\section{Référence électronique}

Jacques Coulardeau, «La culture de la réalité virtuelle», ASp [En ligne], 5-6 | 1994, mis en ligne le 06 décembre 2013, consulté le 10 décembre 2020. URL : http://journals.openedition.org/asp/4045 ; DOI : https://doi.org/10.4000/asp.4045

Ce document a été généré automatiquement le 10 décembre 2020.

Tous droits réservés 


\title{
La culture de la réalité virtuelle
}

\author{
Jacques Coulardeau
}

1 Nous pouvons attaquer le problème par bien des entrées. Par exemple Marshall MacLuhan :

Visual space is uniform, continuous and connected. The rational man in our Western culture is a visual man. The fact that most conscious experience has little

"visuality" in it is lost on him. (1967: 45)

2 C'était le temps où l'on posait le visuel comme non intellectuel, voire dangereux pour la pensée. Il dit encore, cependant:

the book is an extension of the eye... electric circuitry, an extension of the central

nervous system. (1967:34-40)

On sent là une contradiction car le livre (et donc la lecture) fait partie du visuel. Nous sommes cependant au cœur du débat qui va nous tenir pendant quelques pages. La culture de la "réalité virtuelle » ou plus généralement de l'informatique dans sa phase actuelle est une culture visuelle. Il nous faut la définir.

\section{La visualisation comme mode de pensée}

4 Tout commence vraiment avec Einstein, que La Garanderie 1990 a lu :

Je pense très rarement en mots d'ailleurs. Une pensée vient, et je peux essayer de l'exprimer en mots ensuite... Mais qu'est-ce au juste que la " pensée »? Lorsque, à la réception des impressions sensorielles, des images mentales émergent, ce n'est pas encore «la pensée ». Et, quand de telles images se constituent en séquences, dont chaque membre en appelle une autre, cela n'est toujours pas «la pensée». Pourtant, lorsqu'une de ces images se retrouve dans plusieurs séquences de ce type, elle devient - par sa récurrence même - un élément organisateur, puisqu'elle relie entre elles des séquences disparates. Un tel élément est alors un outil, un concept. Je crois, pour ma part, que la transition de l'association libre, ou de la « rêverie ", à la pensée en tant que telle, est caractérisée par le rôle plus ou moins important joué par le «concept ». Il n'est absolument pas nécessaire qu'un concept soit attaché à un signe reconnaissable ou reproductible sensoriellement (le mot); on peut dire en 
revanche que lorsque tel est le cas, la « pensée » devient communicable. (Einstein, Autoportrait, Interéditions, 1980 : 13-14, cité par La Garanderie, 1990) visualiser. Il a besoin ensuite de concevoir des concepts dans cette visualité, des concepts qui sont visuels à nouveau et qui organisent par leur récurrence signifiante le sens de ce qu'il voit. La Garanderie fonde ensuite tout son raisonnement sur cette idée que certains hommes sont des visuels et qu'aucune pensée n'est possible pour eux sans passer par cette étape. Il les oppose aux auditifs ou linguistiques qui, eux, font tout passer par du langage qu'ils se disent dans leur tête. Il n'en reste pas moins que pour les visuels au moins il faut voir ce que l'on pense pour le bien penser. C'est là qu'intervient une première discussion nécessaire que nous devrons trancher dans le cours de cette contribution : le visuel est-iI nécessaire pour tous et représente-t-il un niveau supérieur de pensée?

Howard Rheingold nous emmène alors au Japon :

Japanese writing, based on Chinese-originated kanji pictograms that still retain a pictorial as well as a semantic content, is naturally a visually rich language. People and trees, oxen and rivers, natural forces and recognizable human activities seem to jump out from certain kanji figures when they are pointed out. The act of learning how to express oneself in kanji requires a kind of training of visual thinking skills. American children learn 26 letters, usually written with pencils. Japanese children learn to paint hundreds of pictograms. At least one distinguished Japanese researcher has claimed that because of the pictorial thinking stimulated by kanji Japanese readers use a different part of their brain than readers of alphabetic languages. (1991: 250)

Et encore :

As Dr Kanda explained, kanji writing, based on Chinese pictograms, has a pictorial meaning along with the layers of abstractions that have been added to it. A character might represent a certain phoneme that could be part of a word for computer or canteloupe, but the visual representation of that character still might have a lingering visual reference to a rock or a person. The English alphabet is meaningless on the iconic level. That means that Japanese and English readers use different parts of their brains when they read a language. Reading Japanese characters requires more visual thinking than reading English alphabetic characters, which have no other layers of meaning beyond their strictly symbolic encoding. It made sense to me that this is one of the reasons visual communication is an important goal at ATR (Advanced Telecommunications Research Institute International) and NTT (Nippon Telephone and Telegraph). (p.293)

7 C'est de ces approches et de ces réflexions qu'émerge le concept de Vizthink (pour : Visual Thinking). Cela rejoint parfaitement les recherches les plus classiques sur le cerveau qui expliquent déjà que la lecture ou l'écriture mettent en jeu une zone cérébrale supplémentaire par rapport à la simple parole émise ou reçue, le gyrus angulaire. L'utilisation d'une langue iconique met en cause, donc, une zone visuelle spécifique, du moins si nous en croyons le docteur Kanda. Suivant cette ligne de raisonnement, le visuel dans la communication linguistique permet le développement du cerveau, donc le développement mental.

Une troisième approche, plus psychologique, nous vient de Alan Kay, l'un des développeurs du système Star de Xerox et l'inventeur du concept de Dynabook de l'ordinateur portable. Il part des recherches de Jerome Bruner qui a repris la célèbre expérience de Piaget sur la conservation de la quantité avec l'eau que l'on transvase d'un gros verre bas dans un verre haut et fin. L'enfant traditionnellement dit qu'il y a plus 
d'eau et Bruner a vérifié ce résultat. Mais il a ensuite caché le verre haut et fin et l'enfant a alors revu le gros verre bas et il s'est alors dit: "Mais non, c'est impossible d'où viendrait-elle? ». Bruner a alors révélé à nouveau le second verre et l'enfant est revenu à son idée d'une quantité plus grande. Cela mène ces chercheurs américains à penser qu'il n'y a pas trois étapes successives et exclusives, une étape kinesthétique, une étape iconique et une étape symbolique mais plutôt trois mentalités qui cohabitent toujours ensemble avec des équilibres différents selon le moment et l'activité : une mentalité kinesthétique, une mentalité iconique et une mentalité symbolique. Le génie d'Alan Kay et autres développeurs informatiques, c'est d'avoir inventé le PC et les GuIs. Pour ces machines les trois mentalités sont nécessaires.

The obvious thing seemed that what we should do is try to find some synergies between these mentalities that don't often talk to each other. And the way that you practically realize it in a computer system is you give kinesthetic contact with what is going on by using something like a mouse. You could make that a lot stronger by making the mouse react to the things that you are dealing with. We have mice at Apple where you can feel the weight of the things that you are moving around, which gives you an even closer kinesthetic contact with it. Then you have the icons and the windows, which give a theatrical representation of something underneath that your visual system can recognize almost instantly. And then, finally, you should have a system like NyperCard or SuperCard or something like that, which provides a symbolic underpinning that gives you abstraction in each of these different areas. And that was our rendering of Bruner's educational environment back then. (Kay $1992: 43$ )

9 Nous avons donc là trois approches qui impliquent que le visuel est capital dans le développement mental, mais surtout une troisième qui pose que les technologies modernes des interfaces graphiques est une révolution au niveau de la pensée car elle nie un attendu généralement accepté (Piaget), elle propose un système qui fonctionne sur d'autres lignes, et surtout elle a rencontré un succès inouï auprès du public, alors même que si l'on en était resté à Piaget, cela eût dû être un échec. Ce succès transforme en retour les mentalités et les façons de penser. L'impact d'une telle approche théorique est immense: elle prouve par la pratique sa propre vérité et elle transforme la réalité conformément à ses propres attendus. On touche là à une révolution technologique qui modifie nos façons d'agir, de penser et de concevoir le monde et notre action. Notre pensée en est complètement transformée. Et c'est cette transformation que j'aimerais maintenant analyser.

\section{Technologies capacitantes et amplification de l'intelligence}

10 Le premier concept de technologie capacitante est fondamental et découle directement de ce que nous venons de dire. Il y a, dans le monde des techniques, deux types de techniques. D'abord une technique qui satisfait un besoin concret et précis de l'homme comme le marteau, si on veut. Mais ensuite une technique qui trouve sa finalité dans sa capacité à rendre possible une autre technique, donc qui ouvre une porte à un développement ultérieur, par exemple le chemin de fer pour l'industrie.

An enabling technology is one that makes another technology possible. Some powerful technologies spring into existence when they do because some kind of price or performance threshold has been crossed by one or several enabling technologies. The vacuum tube, for example, was the enabling technology for both 
radio and television, just as the steam engine was the enabling technology for both locomotives and electric dynamos. Dynamos and incandescent bulbs ware enabling technologies for the electrification of the world. Virtual reality based on computers and head-mounted displays has been dreamed of for decades, but had to wait for enabling technologies of electronic miniaturization, computer simulation, and computer graphics to mature in the late 1980s. (Rheingold $1991: 61$ )

11 Je pense qu'il faut élargir également le concept de technologie capacitante et la considérer de deux points de vue. D’une part du point de vue du développement technique pur et là le texte de Rheingold est parfaitement clair. Mais aussi du point de vue $\mathrm{du}$ développement que cette technologie permet, non pas seulement de la technologie en tant que telle, mais des activités humaines. Ainsi une technologie capacitante entraîne un développement extrêmement rapide de domaines importants de l'activité humaine, que ce soit au niveau social (l'industrie par exemple ou les communications) ou au niveau individuel. Que ce soit, encore, directement : l'ordinateur graphique donne à l'individu un pouvoir énorme et quasi instantané moyennant un apprentissage extrêmement rapide et court, et surtout un apprentissage qui peut se faire en cours de route - on peut utiliser l'ordinateur dès le premier instant et on enrichira cette utilisation progressivement. Mais, plus encore indirectement: cela est particulièrement vrai des technologies de la communication. L'utilisateur devient alors un récepteur d'innombrables messages qui lui permettent d'enrichir ses connaissances, sa culture et de se développer personnellement, ce qui deviendra, s'il sait y veiller, un atout dans sa valeur sociale. D'ailleurs celui qui se refermerait totalement sur lui-même et refuserait toute communication moderne deviendrait un être a-social voire anti-social. Prenons un dernier exemple. Lorsque le CD est apparu, on l'a voué à l'échec. Il a réussi, prouvant ainsi que les hommes étaient prêts à recevoir un son de meilleure qualité. Il a d'autant plus réussi que ses utilisateurs ne veulent plus l'abandonner et qu'il a même permis d'apprécier la valeur particulière du son-vinyle antérieur, son qui est recherché aujourd'hui par des puristes ou des collectionneurs. Mais plus encore on s'est moqué de la bande passante des CD qui montait jusqu'à 21000 hertz. Or on s'est aperçu depuis que beaucoup d'entre nous ne sont pas sourds à ces sons. Il reste à faire des enquêtes précises pour avoir des chiffres honnêtes, mais cette technologie, qui a développé l'audition des hommes dans la bande passante reconnue, révèle et peut-être développe l'audition des hommes au-delà de cette même bande, permettant ainsi à des recherches nouvelles, jusque là totalement non signifiantes, de se faire jour et de se développer. Voilà ce qu'est une technologie capacitante.

12 Le deuxième concept est celui de l'« amplification de l'intelligence ». Là encore Rheingold est très clair. Certaines technologies nous permettent d'élargir notre champ intellectuel et donc de donner à notre intelligence des capacités nouvelles. On est loin de la simple vitesse de calcul d'opérations complexes. On est bien plus haut.

'When you talk to Fred Brooks,' Robinett (a leading infonaut at UNC) told me, 'be sure to ask him about IA - intelligence amplification. That's why I came here. It dovetails perfectly with my take on computer graphics. I want to use computers to expand human perception. We have certain built-in senses such as vision, hearing, and smell, but there are many phenomena which are completely imperceptible to us. Some examples are X-rays, radioactivity, electricity, and the inside of opaque objects. You could say that we lack the senses to perceive these things. But using electronic sensors and computer displays, we can make these imperceptible phenomena visible. Or audible or touchable. I call the apparatus for doing this a sensory transducer. "One thing we're working on here at UNC is hooking up an ultrasound scanner to the head-mounted display, so that instead of looking at an 
ultrasound image on a monitor screen, a doctor could put on the headset and see directly into the living tissue. This would be, in effect, a synthetic sense similar to Superman's X-ray vision.' (Rheingold $1991: 25-26$ )

On retrouve ici Ia discussion de notre premier point, à savoir la dimension visuelle nécessaire à la pensée. Si effectivement ces nouvelles technologies de Réalité virtuelle sont capables de nous faire voir, et qui plus est d'une façon nouvelle, plus prégnante, plus directe et proche, des phénomènes que nous ne sommes pas naturellement capables de voir, cela va nous permettre d'augmenter la matière première de notre intelligence, et donc de développer notre intelligence. De nouvelles méthodes de travail vont apparaitre, de nouvelles méthodes de pensée aussi. Il faudrait ajouter à cela les ordinateurs neuronaux et surtout les recherches nouvelles sur des langages plus performants. Nous avons aujourd'hui à la disposition de notre intelligence des outils qui sont capables de nous emmener dans des mondes inconnus et nous en serons complètement transformés.

Pour conclure ici, il faut voir que l'inverse est possible. Je ne prendrai qu'un exemple, celui de la couleur. Pour un statisticien, un univers tridimensionnel comptant quelques dizaines de couleurs est parfaitement dominable. Il travaille avec des outils de ce genre tous les jours et son travail en est enrichi. Mais qu'en est-il pour un opérateur de robot industriel qui doit saisir des messages de façon parfaite, très rapide et agir dans l'instant qui suit? On s'aperçoit qu'on ne peut pas jouer sur beaucoup de couleurs : cinq sont le maximum, et le bi-dimensionnel est plus sûr :

Restrict the number of colors on a coded display to about 12, or as few as five in critical tasks.. . Navigation through a three-dimensional perceptual color model may be fine for a research statistician, but it may be insppropriete for a machinist in a factory. (« The proper use of color »1991 : 841S-39)

Les hommes et les situations, dans lesquelles ils se retrouvent, ne sont pas égaux ou égales. Cela ne veut pas dire que l'ouvrier concerné n'est pas capable d'apprécier Rembrandt, mais cela veut dire qu'une éducation particulière est nécessaire pour que la sécurité de l'utilisation de couleurs fines soit garantie dans des situations d'urgence. On a parlé de technologies capacitantes et d'amplification de l'intelligence. Cela est vrai pour tous, pour la société dans son ensemble, mais cela est inégal pour les individus et inégal pour les situations. Le progrès bien sûr a l'art de montrer les limites des uns et des autres.

\section{Interactivité}

Le monde de la Réalité virtuelle est un monde à part.

It should be user delightful. (Pimentel \& Teixeira $1993: 155$ )

C'est là un concept neuf que certains critiquent véhémentement, l'accusant même de provoquer l'épilepsie de certains sujets. Il nous faut cependant l'explorer avec un tant soit peu de précision.

Creating virtual worlds is a new field full of challenges and rewards. Largely unexplored, it beckons the adventurous, the curious and the inventive mind with its flexibility and power. In a scientist's hands it becomes a tool as basic as a microscope. To a teacher it suggests new ways of educating children and adults. For many people it's simply a great new form of entertainment. And for artists it inspires new forms of art, ones in which there's no longer a separation between art and audience. (Pimentel \& Teixeira $1993: 144$ )

8 La première des remarques à faire est que ce monde, qui se réalise aujourd'hui dans les jeux électroniques, est un monde qui développe de façon extrêmement dynamique la 
vision de l'espace et du temps. Patricia Greenfield, de UCLA, a expliqué dans un colloque à Roubaix sur les jeux électroniques et l'interactivité en novembre 1993, comment un joueur ne peut pas réussir sérieusement dans le moindre jeu électronique s'il ne se construit pas mentalement une carte complète de l'espace dans lequel il entre et du temps qui l'accompagne. Il doit voir toutes les pièces, toutes les sorties et toutes les entrées, tous les pièges et toutes les primes. Il doit savoir la disposition de tous les lieux et de tous les ennemis avant même de pouvoir réussir. Sa stratégie est toujours la même: faire le jeu une première fois pour découvrir les secrets de ce monde, puis le faire pour gagner. Il y a là un développement mental énorme pour un enfant qui peut ainsi démultiplier sa vision de l'espace et du temps et la représentation mentale qu'il en a.

Plus encore, ces jeux sont en train de devenir interactifs. On distingue plusieurs niveaux d'interactivité. D'abord l'interactivité de sélection qui ne laisse pour toute liberté que celle de zapper d'une chaîne à une autre. Puis l'interactivité parallèle sous la forme de jeux télévisés qui fonctionnent avec la possibilité du spectateur de prédire la suite d'un programme et de gagner des points si la prédiction est juste. Puis il y a l'interactivité contrainte comme dans Hugo et Télétennis. Mais là encore l'interactivité ne permet pas de changer les paramètres, simplement de se glisser dans un personnage et de suivre sa trajectoire. On nous propose ensuite et enfin l'interactivité dite totale, celle des jeux électroniques, qui consiste à jouer vraiment et donc à utiliser le programme à ses propres fins, bien que les fins soient le plus souvent déterminées par le programme qui reste pour l'essentiel non changeable. On remarque cependant que les jeux de stratégie comme SimCity ou SimEarth donne au joueur le pouvoir de créer lui-même une ville ou de gérer la terre. Le programme est bien sûr fixe, mais le joueur part de rien et il peut faire ce qu'il veut. On peut imaginer un autre niveau qui serait encore plus interactif et qui permettrait soit au joueur de changer certains paramètres du programme, soit au programme de s'adapter aux comportements de jeu du joueur et de changer à sa propre initiative en fonction de ce comportement, rendant ainsi les choses plus difficiles, plus « customized ». C'est ce dernier niveau que la Réalité virtuelle vise et permet d'atteindre. On entre dans le monde artificiel et on peut réagir sur le programme qui réagit lui aussi et s'adapte. On n'est certes pas Dieu, mais on est un de ses anges.

On a alors dans ces jeux un environnement qui est « responsive ». À ce moment-là, la psychologie du joueur change, car il joue presque à armes égales avec le programme.

Unlimited freedom is the ultimate responsibility... Virtual reality allows the user unique capabilities. (Pimentel \& Teixeira1993 : 155)

La réalité virtuelle devient alors un théâtre dans lequel nous sommes un acteur qui tient aussi bien les ficelles du jeu que celles de la technique. Il est à la fois acteur, machiniste, technicien et metteur en scène. Le pouvoir est énorme. Le jeu se contient entièrement. Le joueur est coupé du monde extérieur et il est entièrement contenu dans son jeu, dans sa partie, dans l'univers qu'il crée en s'y glissant. "The performance is the thing » (op. cit. : 57)

On peut alors commencer à concevoir la psychologie d'un tel jeu. William Bricken (State University de Washington) a tenté de la résumer en quelques éléments :

Psychology is the physics of VR.

Our body is our interface.

Knowledge is an experience.

Data is in the environment.

Scale and time are explorable dimensions.

One experience is worth a trillion bits.

Realism is not necessary. » (In Pimentel \& Teixera 1993 : 160) 
diront que c'est là une psychologie de la fuite en avant. Il s'agit cependant d'une expérience qui est unique pour des masses de gens dans l'histoire de l'humanité. C'est l'expérience du chercheur scientifique le plus avancé. Il est seul face à sa matière. Il n'y a plus aucune règle. Il doit tout inventer et tout découvrir et il ne connaît rien de ce qu'il va découvrir. Il est le suprême Léonardo Da Vinci devant sa toile blanche ou devant son papier. Il peut tout, sauf ce que ses propres limites physiques l'empêchent de faire. L'utilisateur de Réalité virtuelle va encore plus loin, car il ne connaît même pas les limites de la matière. Il peut inventer des matières nouvelles et il peut s'y plonger totalement. Il a alors un sentiment absolu de liberté et de puissance. Il est Dieu. Il a au bout de ses doigts des forces qui ne lui échappent pas. Il est le maître de l'univers. Il a le langage verbal à sa disposition. Il a le langage visuel à sa disposition. Il a le langage musical à sa disposition. Il a le langage textuel à sa disposition. Il a enfin les langages graphiques et de l'animation à sa disposition. Au commencement était le Verbe ; et aujourd'hui le jeu de Réalité virtuelle qui sera dans nos boutiques d'ici quatre ou cinq ans donne la puissance de ce verbe divin à l'utilisateur. Comment ne pas se sentir emporté par une force qui vous investit et rayonne de vous. Nous sommes dans un autre monde.

main il l'est avec sa propre psychologie de départ. Il peut donc créer un monde humain. Il peut aussi créer un monde barbare. Il peut retrouver une autre force, celle des SS qui tuaient par plaisir et qui faisaient souffrir par lassitude. Il peut se donner les «plaisirs » de tuer, assassiner, massacrer, torturer. Cette remarque montre combien ces jeux sont neutres par rapport à une autre psychologie, plus fondamentale, celle que le joueur a quand il entre dans le jeu. Stephen King a donné une vision assez pessimiste de ces jeux dans Le Cobaye (The Lawnmower Man). En fait le joueur revit toutes ses frustrations et toutes ses ambitions, tous ses désirs d'amour et de mort et il peut leur donner absolument libre cours. On peut donc entrer ici dans un monde infernal.

Doit-on interdire cela sous prétexte que certains vont transformer les jeux en château de Dracula? Non. Cela pose la nécessité d'assurer à chacun un équilibre mental et psychologique complet avant même qu'il entre dans ces mondes virtuels. Mais comment être sûr que l'individu est prêt? On ne peut pas le savoir. Il faut donc laisser le monde se développer et assurer une politique d'accompagnement qui permettra à chacun de trouver le plaisir sans que cela se fasse par le développement et la satisfaction, même symbolique, des instincts les plus bas. Mais comment ne pas voir qu'aujourd'hui déjà tant et tant de magazines et de livres ne répondent qu'à la nécessité pour certains de satisfaire de tels instincts ? Est-ce la faute de la Réalité virtuelle si l'homme est si imparfait?

Aujourd'hui l'homme peut jouer à Dieu, et il est si loin de Dieu. Autrefois il ne pouvait pas y jouer. Il pouvait se faire artiste et l'imaginer. Il pouvait se faire bourreau et réaliser ses instincts de mort. Mais aujourd'hui tout un chacun, et non pas quelques-uns vont bientôt pouvoir faire cela. On ne sait pas quelles seront les conséquences de ces jeux, mais on sait une chose: on ne peut pas les interdire ni les empêcher, car on ne peut pas dans notre monde moderne empêcher la circulation des œuvres de l'esprit, qui dans ce cas précis sont neutres, puisque les tendances négatives, comme les tendances positives, seront introduites dans le jeu par le joueur. 


\section{Où est encore la réalité ?}

27 Le langage de l'informatique est en pleine mutation. Il y a d'abord le langage de programmation qui est un langage tout à fait particulier. Il allie du langage naturel, le plus souvent des mots ou des expressions et une syntaxe abstraite qui correspond à la logique de fonctionnement de ce langage, de la programmation et des machines. Il serait intéressant de travailler en linguiste les divers langages disponibles, mais ce sont des langues étrangères. Ces langages fonctionnent selon des normes et suivant des lignes qui sont déterminées par le hardware et le software de ces machines, donc loin des langages naturels. Ce sont des métalangages. L'utilisateur doit utiliser ces langages pour sa communication avec la machine.

Puis il y a eu les interfaces utilisateurs. Les métalangages ne changeaient pas mais le langage que l'utilisateur employait, lui, changeait. L'utilisateur était confronté à des fenêtres et des menus qui parlaient normalement, c'est à dire en langue naturelle, à l'origine l'anglais, et aujourd'hui dans un ensemble de langues variées. Ces fenêtres donnent des instructions, transmettent des messages et surtout proposent des choix sous forme de menus qui eux aussi parlent en langue naturelle. Cependant ces menus comportent des termes de métalangue. Il est nécessaire de comprendre cette métalangue. C'est essentiellement du lexique simple (mots simples) ou du lexique complexe (mots composés). Complexe, donc, en fonction des opérations proposées, mais ce n'est que du lexique. Il est vrai que très peu de développeurs ont jugé bon de faire de vrais dictionnaires de ces termes. Il existe des dictionnaires d'informatique anglais, français ou bilingues, fort bien faits, mais il n'existe pas de dictionnaire des instructions d'ordinateurs. Même Macintosh ne semble pas en avoir fait un. À ce moment-là, on avait alors encore un problème de communication avec l'utilisateur qui cependant ne devait plus que choisir et cliquer.

29 C'est alors que les GUIs sont apparues. Au lieu d'avoir des menus donnant des choix entre des opérations nommées, on est passé à des menus d'opérations représentées par des icônes. Généralement les deux sont associés: le nom et l'icône. À ce moment-là, l'utilisateur communiquait avec l'ordinateur par l'intermédiaire de ces icônes, en choisissant puis cliquant. Le langage n'est plus alors un handicap. Quand on travaille sur un certain nombre de logiciels, raison de plus d'une seule entreprise, on retrouve de nombreuses icônes communes. Cependant ces icônes ont été déposées et donc la célèbre corbeille ou poubelle de Macintosh ne peut être utilisée par les autres marques. Mais comme on ne mélange que peu les standards de logiciels, cela ne pose pas problème, sauf quand on passe de Macintosh à IBM. Nous en sommes là.

La communication de l'utilisateur, disons de surface se fait par des barres de menus, des menus, des icônes et des messages éventuels de réponse. L'utilisateur superficiel ne va pas plus loin. L'utilisateur plus profond remonte, lorsque c'est possible (dans les PC) au DOS et passe alors au métalangage instructionnel sans passer à la programmation. Mais il touche là déjà à ce métalangage. L'utilisateur spécialiste ou professionnel, lui, va être capable de programmer l'ordinateur et donc d'intervenir directement dans le DOS pour changer les paramètres, modifier les fonctionnements, bref configurer sa machine à sa mode. Il peut, s'il est un informaticien programmeur être amené à écrire des programmes nouveaux dans un métalangage et ensuite à les importer sur tel ou tel DOS pour les appliquer. 
31 La prochaine étape est le passage à l'ordinateur vocal qui sera capable de prendre ses instructions vocalement et de répondre de la même façon. Cela est indispensable pour les malvoyants par exemple, et aussi pour les utilisateurs qui ont des problèmes avec la navigation dans un univers graphique de ce genre. Cela pose des problèmes de tolérance de variation vocale et surtout de tolérance de variation linguistique. Il faudra employer des termes standardisés, donc une métalangue qui ne pourra pas être iconique.

Notons pour finir que les DOS les plus francisés continuent largement à parler en anglais dans tout ce qui est instructions. Ainsi diskcopy reste diskcopy et en anglais, même sur un DOS francisé pour grand public. Il reste donc là un problème de langue étrangère.

Mais j'aimerais attirer l'attention sur le fait que le langage de l'ordinateur intervient directement sur le fonctionnement mental et intellectuel. Comme tout métalangage, le métalangage de l'ordinateur est structurel et donc il structure le fonctionnement du cerveau de l'utilisateur. Des études précises doivent être faites. Mais de nombreux utilisateurs en tombent dans un langage que je dirai déictique : «Tu appuies là, puis là, et ensuite là. Tu choisis ça et tu obtiens çà. Tu vas dans la fenêtre d'en haut et tu prends cette instruction et tu cliques et tu obtiens ça... ». À ce niveau, il y a régression dans le langage, et les GUIs prêtent le flanc à cela. On observe une verbalisation minimum des icônes et rien d'autre. En français on a même une verbalisation qui semble aller dans deux sens : d'une part des noms français bricolés et que seul le petit groupe qui travaille dans tel ou tel atelier peut comprendre; d'autre part l'importation en français de termes anglais non traduits mais francisés dans la prononciation. Dans ce dernier cas, il n'y a aucune compréhension de ce que le terme anglais veut dire. On en est revenu à la simple désignation référentielle directe. Il y a régression en deçà du concept qui lui ne peut se développer que sur la base du mot et donc de son sens, de sa morphologie, de son histoire même.

Plus encore, et cela au niveau plus avancé des programmeurs, il y a réduction de tout processus de pensée à un langage binaire linéaire d'une machine de Türing avancée. Tout phénomène est réduit presque automatiquement à des suites d'opérations binaires, même quand de toute évidence le phénomène étudié n'est pas binaire. L'exemple le plus simple est celui de la coordination en langue qui produit d'abord «Paul+et » puis ensuite " (Paul +et)+Pierre». Cela empêche de rendre compte de l'inversibilité des deux termes nominaux ou autres de la coordination. Ce phénomène a une force telle qu'il en devient bloquant au niveau même de la conception. Et il est effectif que les machines actuelles ne sont pas capables de rendre compte de phénomènes non binaires, sinon par des instructions lourdes et donc coûteuses en mémoire et en programme.

Mais j'aimerais avancer sur un dernier point. La Réalité virtuelle va beaucoup plus loin dans l'éloignement de la "matière ». Elle crée un univers plurisensoriel entièrement artificiel, c'est à dire non tiré de la réalité matérielle extérieure, ou du moins qui peut fonctionner de cette façon. L'utilisateur vit alors dans un monde artificiel. Si c'est un musicien, il va créer des sons et des harmonies nouvelles. Bien. Il étend le monde musical. Si c'est un artiste, il va être capable d'imaginer des mondes graphiques nouveaux, des couleurs nouvelles, etc. Il va ainsi développer le monde pictural ou théatral ou cinématographique selon le domaine où il travaille. S'il est chorégraphe, il peut imaginer des pas nouveaux, des mouvements nouveaux et ainsi développer le monde de la danse. Mais s'il joue avec ce monde, s'il s'y plonge pour le simple plaisir de l'aventure, que devons-nous penser du monde dans lequel cet utilisateur se trouve? Monde réel ou monde artificiel ? La ligne de partage a bougé. 
De toute évidence ce monde est matériel puisqu'il est produit par des machines et qu'il est reproductible. Mais voilà une matière qui est issue non pas de rien, mais de quelque chose qui n'est pas la réalité matérielle tangible. Ce monde est donc imaginaire et irréel. Certains vont même jusqu'à dire que l'on peut se trouver accroché à de tels mondes, comme si c'était une drogue. On rejoint le concept de paradis artificiel. Or pour moi, il s'agit bien d'un nouveau domaine de matérialité au sens le plus physique du terme. Je ne vais donner que quelques raisons.

1. Il est produit par des machines.

2. Il peut être produit par n'importe qui, mais en tout cas par un humain.

3. Il peut être reproduit.

4. L'utilisateur investit ses pulsions personnelles dans ce monde qu'il a créé. Tant ses pulsions positives (libido) que ses pulsions négatives (instinct de mort). Ces pulsions sont matérielles, à moins de nier encore Freud, Reich, Jung et Lacan.

5. Ces machines et ces programmes sont produits par un travail qui a un statut et une rémunération et donc une valeur : des graphistes, des informaticiens, des artistes divers, etc.

6. Ces machines et ces programmes ont une valeur mercantile sur un marché parfaitement défini et régulé : ce sont des biens marchands.

7. Ces machines peuvent permettre à l'utilisateur d'enrichir sa vision du monde soit en lui permettant de voir l'impossible, soit en étendant le possible vers des impossibles, qui par là même deviennent possibles. Cela ouvre la porte à d'innombrables applications concrètes: médecine, physique, mathématique, autres sciences exactes; technologies diverses et en premier lieu de la communication ; toute science qui utilise des modèles.

Il n'en reste pas moins que certains vont parler de ces machines comme de la nouvelle frontière de l'aventure. D'autres comme une drogue dangereuse car elle vous coupe du réel. D'autres encore comme un artifice diabolique qui révèle et amplifie les tendances négatives de l'homme. Tout tourne autour de la définition du réel. Soit le réel n'a pas de rivages - selon le terme d'Aragon - et donc on peut aller au-delà de ce qu'on connaît. Soit le réel est le matériel tangible et aller au-delà n'est qu'artificiel car menant à quelque chose qui n'existe pas et ne peut pas exister. Le film More a montré où cela peut mener. Soit le réel n'est que ce qui est acceptable pour la morale humaine, le reste étant le mal que l'on doit nier. Qu'on relise l'Areopagitica de Milton!

\section{Conclusion}

Nous venons de poser des positions éthiques. Nous en avons identifié trois. Il risque d'y en avoir d'autres. Il n'en reste pas moins que ces machines sont déjà là et que bientôt des millions d'utilisateurs seront dessus. Si ce n'est pas matière réelle, qu'est-ce? Les utilisateurs vont en tirer profit au niveau de leur intelligence, de leur pensée, de leur pouvoir sur eux-mêmes et sur les autres. Ils en seront augmentés, grandis, développés. Et cela est encore une matière réelle qu'il faut d'urgence utiliser. Mais ces machines vont libérer chez l'utilisateur toutes ses tendances profondes. Elles sont donc un outil de liberté, positif ou négatif en fonction des tendances profondes de l'homme. Cela est parfaitement matériel, mais comment éviter les dangers que ce phénomène porte en lui ? C'est la question cruciale : comment domestiquer ces nouvelles technologies qui libèrent des forces jusque là dominées? Comment domestiquer ces forces qui se libèrent avec ces nouvelles technologies? Une chose est sûre : tous les discours de rejet sont inacceptables. Ces technologies ne sont pas le diable, elles ne créent pas le mal. Elles ne sont que des 
outils qu'il nous faut apprendre à utiliser. Le phénomène nouveau c'est que des millions de gens les auront bientôt avant même que nous sachions nous en servir. On est assez loin du marteau, mais assez près de Dieu. De là à en devenir déiste, il n'y a qu'un pas, que l'on ne peut pas franchir, puisque bientôt nous serons des millions de dieux vivants et respirants. Voilà sans doute la plus belle transformation du siècle prochain.

\section{BIBLIOGRAPHIE}

Kay, Alan. 1992. « Mind nelding ». Byte Outlook.

King S. 1992. The Lawnmower Man. New York : Allied Vision Lane Pringle.

La Garanderie A. 1990. Pour une pédagogie de l'intelligence. Paris : Bayard Éditions.

La Garanderie A. 1991. La motivation, son éveil, son développement. Paris : Bayard Éditions.

MacLuhan M. 1967. The Medium is the Massage. New York: Bantam Books.

Pauloss J. A. 1988. Innumeracy, Mathematical Illiteracy and its Consequences. New York : Hill And Wang.

Pimentel K. \& Teixeira K. 1993. Virtual Reality through the New Looking Glass. New York : Intel Windcrest Macgraw-Hill.

Rheingold B. 1991. Virtual Reality. New York : Simon and Schuster.

«The proper use of color ». Byte, December 1991.

\section{RÉSUMÉS}

La Réalité virtuelle révèle l'importance de la pensée visuelle dans le monde moderne. Devonsnous développer la pensée visuelle chez tous les apprenants? La réalité virtuelle est une technologie capacitante pour la communication et est la source d'une importante amplification intellectuelle. Elle est d'autant plus importante qu'elle devient interactive. Dans ce monde, la psychologie devient essentielle et centrale. Nous devons poser que la réalité virtuelle et ses créations sont matérielles. Il en découle la question de la nature de la réalité.

Virtual Reality reveals the importance of visual thinking in our modern world. Must we develop visual thinking in all learners? Virtual Reality is an enabling technology to modern communication and it is the source of an important intelligence amplification. It is all the more important as it is becoming interactive. In this world psychology becomes essential and central. We must state virtual reality and its creations are material. Hence where is reality?

\section{INDEX}

Mots-clés : interactivité, réalité virtuelle, technologie

Keywords : interactivity, technology, virtual reality 


\section{AUTEUR}

\section{JACQUES COULARDEAU}

Jacques Coulardeau enseigne au Lycée Jean Rostand, Roubaix et en LEA à l'Université de Lille 3, Roubaix.dondaine@orange.fr 